\title{
Tangence
}

\section{Rhétorique de la critique littéraire africaine}

\section{Josias Semujanga}

Numéro 51, mai 1996

Paradigmes critiques

URI : https://id.erudit.org/iderudit/025906ar

DOI : https://doi.org/10.7202/025906ar

Aller au sommaire du numéro

Éditeur(s)

Tangence

ISSN

0226-9554 (imprimé)

1710-0305 (numérique)

Découvrir la revue

Citer cet article

Semujanga, J. (1996). Rhétorique de la critique littéraire africaine. Tangence, (51), 81-97. https://doi.org/10.7202/025906ar d'utilisation que vous pouvez consulter en ligne.

https://apropos.erudit.org/fr/usagers/politique-dutilisation/ 


\section{Rhétorique de la critique littéraire africaine Josias Semujanga}

\section{Critique littéraire, un discours doxologique}

Cette étude qui vise à l'élaboration d'une réflexion sur la rhétorique de la critique littéraire africaine et son application sur les œuvres particulières, par exemple le roman, poursuit également deux objectifs spécifiques: 1) identifier les différents traits génériques relevés par les essais critiques comme étant constitutifs de l'"africanité " ou de l' européanité " des œuvres africaines et 2) déterminer comment ils instruisent le procès esthétique et axiologique du discours critique. Sur quoi porte ce double procès? Deux hypothèses sont possibles. 1) Le discours critique littéraire traite du processus de création littéraire et décrète ce que doit être l'écriture d'une ouvre particulière; 2) c'est à partir des stratégies énonciatives et argumentatives qui lui sont propres que le critique intègre des éléments liés aux genres littéraires et des éléments liés au contexte d'époque (africanité ou européanité).

Sans entrer dans les détails sur la problématique de la notion de discours critique littéraire, trop complexe pour être abordée dans le cadre de cet article, rappelons que le discours de la critique littéraire peut être considéré comme un discours doxologique ${ }^{1}$ qui, ainsi que le dit justement Jean Terrasse au sujet de l'essai, est "le produit d'une tension entre deux désirs apparemment contradictoires: décrire la réalité telle qu'elle est en elle-même et imposer un point de vue sur elle" ${ }^{2}$. Il n'y a pas seulement l'énoncé neutre d'un jugement mais l'implication du sujet critique qui marque son dis-

1 Le discours critique a été étudié par de nombreux chercheurs. Pour les différentes typologies en cours, celle de Marc Angenot me paraît plus générale et plus précise. Après avoir souligné les difficultés de décrire l'essai comme genre constitué, faisant l'objet de règles et de contraintes a priori, présentant un réseau de marques et de signes spécifiques, l'auteur a quand même proposé, dans son ouvrage La parole pampblétaire. Contribution à la typologie des discours modernes, Paris, Payot, 1982, une typologie générale de la littérature d'idées. Dans cet article, j'utilise certains des concepts opératoires définis par l'auteur comme le discours doxologique, cognitif, agonique, etc.

2 Jean Terrasse, Rhétorique de l'essai littéraire, Montréal, Les Presses de l'Université du Québec, 1977, p. 129. 
82

cours. Autant dans l'ordre de son discours que dans son discours même, le critique littéraire cherche à susciter une prise de position sur la littérarité des ouvres qu'il dévoile aux lecteurs et à agir, par des procédés rhétoriques divers, sur eux. À la limite, le critique littéraire ne constate pas la littérarité d'un texte qui préexiste à son intervention discursive. Il énonce avant tout la volonté qu'il en soit ainsi. Par là il réalise non seulement la projection de sa connaissance de la littérarité des ouvres, mais la donne aussi sous sa forme jugée. L'important n'est plus de lire ce qui est littéraire, mais de rendre littéraire ce qui est lu.

C'est pour cela que le discours de la critique littêraire s'articule autour de deux isotopies fondamentales et solidaires dans la constitution de la spécificité de ce type de discours. Liêe aux figures à connotation littéraire (genres, motifs, thèmes, formes, etc. ), l'isotopie littéraire a pour fonction de rattacher le discours critique à un lieu appelé "littérature ". Elle est également liée à l'isotopie référentialiste et idéologique qui articule le discours critique à un ancrage historique et géographique. Ces deux isotopies fonctionnent comme des indices qui opposent la critique littéraire à d'autres types de critiques (historique, sociologique). Argumentatif et polémique, le discours critique littéraire présuppose un débat sur la littérarité et la nationalité des œuvres. Dans son élaboration le discours critique présuppose l'existence de la littérarité en tant que schéma - constitué d'un ensemble de formes et figures à connotation littéraire - dont les œuvres particulières attesteraient l'usage. Ce qui, également, laisse sous-entendre que les ouvres en tant que procès sont structurellement marquées par leur appartenance à la littérarité. Pour être littéraire, toute œuvre présuppose l'existence de la littérarité et d'une compétence particulière qui la réalise. C'est le procès esthétique du discours critique. Par ailleurs, il est aussi traversé par ses positions idéologiques qui justifient, entre autres, le choix des traits formels correspondant à l'ethos du sujet critique au sujet de la nationalité/universalité des textes. C'est le procès axiologique. Ce mélange complexe, composé des isotopies littéraires et des isotopies référentielles et idéologiques, permet de classer tel ou tel discours comme critique littéraire.

Ces réflexions conduisent à nous interroger sur l'existence d'un discours critique littéraire pouvant à la fois faire l'objet d'études auquel il est possible d'appliquer une grille de lecture pour analyser des positions des sujets critiques et être un moyen 
pour proposer une autre façon de lire des œuvres littéraires. C'est dans cette double perspective méthodologique que mon étude portera l'analyse sur la critique africaine et se terminera sur une proposition de lecture du roman africain.

\section{Critique africaine : états des lieux}

Depuis quarante ans, le discours de la critique littéraire africaine est caractérisé par un problème de méthodes et de positions idéologiques. En ce sens elle est traversée globalement par deux tendances. La première, "afrocentriste", affirme que la spécificité des cuvres africaines vient du fait que celles-ci sont une continuation de la parole artistique de la tradition orale. La deuxième, "européocentriste", soutient au contraire que les œuvres africaines en langues européennes prolongent plus ou moins les littératures des anciennes métropoles en Afrique ${ }^{3}$. Ce schéma, mieux qu'un autre, permet de regrouper, me semble-t-il, les différentes tendances critiques sans oublier les risques d'arbitraire que pourrait comporter un tel classement.

Le choix du roman s'explique par le fait que ce genre occupe, plus que tout autre, une place dominante aussi bien par le nombre des ouvres produites que par la multiplicité esthétique adoptée par des écrivains. Dans la mesure où le genre romanesque possède une caractéristique d'“introduire dans son entité toutes espèces de genres, tant littéraires (nouvelles, poésies, poèmes, saynètes) qu'extra-littéraires (études de mours, textes rhétoriques, scientifiques, religieux, etc.) " 4 , chaque romancier lui imprime sa propre vision esthétique tout en le situant par rapport à d'autres esthétiques génériques. De la sorte, le travail du romancier africain consiste à aménager dans le genre romanesque une cuvre particulière susceptible d'emprunter ses formes et ses thèmes tout aussi bien à la tradition romanesque européenne qu'aux récits africains. Cette situation que d'aucuns qualifient de "paradoxale" 5 , suscite un débat portant sur le sempiternel

3 Notons que les deux tendances sont séparées pour les besoins méthodologiques, sinon les deux s'inscrivent dans la vision "européocentriste", comme nous allons le voir.

4 Mikail Bakhtine, Estbétique et théorie du roman, Paris, Gallimard, 1978, p. 41.

5 Dans son article, "Les paradoxes du roman africain", Présence africaine, $\mathrm{n}^{\circ} 139,1986$, p. 74-87, Mohamadou Kane affirme que les caractéristiques 
84

problème de la spécificité de la littérature africaine et de l'influence européenne. Dans les pages qui suivent, je vais analyser tour à tour les deux tendances opposées - afrocentriste et européocentriste - qui se partagent l'espace du discours critique littéraire africain.

\section{Critique " afrocentriste", un discours agonique.}

Le discours critique afrocentriste s'édifie selon une double stratégie: démonstration de l'africanité des cuvres et réfutation d'une thèse adverse: la critique européocentriste. Celle-ci lui sert de contre-discours antagoniste avec lequel il entre en dialogue. En cela, il est un discours agonique. Cette thèse de l'africanité, qui est une vision téléologique et essentialiste dont l'aspect pratique est la valorisation des cultures et civilisations du continent africain, est soutenue par de nombreux critiques. Sa rhétorique est sous-tendue par une quête: inventer une littérature régionale correspondant au projet identitaire du continent noir ${ }^{6}$. Depuis un vingtaine d'années, elle a donné lieu à des ouvrages si innombrables qu'on ne saurait les analyser de façon exhaustive. Heureusement, ils ont tous un air de famille basé sur la quête de l'originalité littéraire africaine. À l'intérieur de cette tendance deux courants peuvent être distingués.

Représenté de façon magistrale par Janheinz Jahn avec son ouvrage Muntu: l'bomme africain et la culture néo-africaine ${ }^{7}$, le premier courant utilise les méthodes anthropologique et philoso-

spécifiques au roman africain, qui le distinguent du roman occidental, se situent au niveau de l'intrigue et de la voix narrative. Aussi, l'auteur se demande-t-il si le concept de roman peut encore servir à désigner valablement les récits africains. Au contraire, je pense que, loin de l'exclure du genre romanesque, ces "paradoxes" du roman africain indiquent justement que le roman est un genre qui s'adapte à toute situation culturelle nouvelle. Ce faisant, le romancier africain ne fait que ce que tout romancier fait: réadapter les traits génériques existants à sa propre vision esthétique.

6 À ce propos l'étude de Nicole Fortin, qui montre comment toute la rhétorique des revues savantes québécoises (1965-1975) est d'inventer une littérature répondant au projet nationaliste du Québec, s'applique très bien au projet de la critique littéraire afrocentriste. Cf. Une littérature inventée. Littérature québécoise et critique universitaire, 1965-1975, Sainte-Foy, Presses de l'Université Laval, 1994.

7 Janheinz Jahn, Muntu: l'bomme africain et la culture néo-africaine, Paris, Seuil, 1975 [1958]. 
phique et prône l'unanimisme de la spécificité africaine. Vers la fin des années cinquante, alors que la littérature africaine était encore à ses débuts, Jahn précisait déjà le devoir-faire du critique et le devoir-être d'une ouvre africaine: "La science littéraire doit découvrir en quoi consiste cette africanité [...]. Il faut chercher quels topoï, quelles idées et quelles caractéristiques de style ont ou n'ont pas leur origine dans des traditions et des civilisations strictement africaines [...]. (p. 17-18). De l'étude de Jahn, retenons trois brèves remarques. 1) En magnifiant l'originalité africaine, l'auteur a ouvert la piste de la comparaison des ceuvres africaines avec les récits de la tradition orale et il a fermé celle de la comparaison avec les littératures européennes au point qu'il a établi une frontière entre la littérature orale et la littérature écrite: "[...] une littérature qui ne témoigne d'aucune influence européenne et qui n'est donc pas écrite n'appartient pas à la littérature néo-africaine mais à la littérature africaine traditionnelle. La frontière entre les deux est facile à tracer: c'est la frontière entre la littérature orale et la littérature écrite. (p. 16). 2) Sa méthode critique, qui est un mélange d'ethnologie, de philosophie et d'histoire, est un outil moins efficace dans l'analyse des textes littéraires. 3) Enfin, constitué essentiellement de textes poétiques, son corpus permet difficilement d'extrapoler les résultats à d'autres genres littéraires comme le roman.

Obnubilé par la question de la continuité entre les récits de la tradition orale et le roman ainsi que celle de l'originalité des textes africains, Mohamadou Kane poursuit, dans son ouvrage Roman africain et tradition ${ }^{8}$, l'héritage de Jahn tout en ouvrant une piste qui sera suivie par de nombreux critiques du roman africain, à savoir que l'originalité du roman africain réside dans le fait qu'il est une continuation des textes oraux de la tradition orale: "[...] l'originalité du roman africain doit être cherchée plus particulièrement dans ses rapports avec les formes de la littérature orale [...]. Une plus grande attention aux rapports, aux liens de continuité des littératures orales et écrites permet de mieux comprendre les problèmes du roman africain." ${ }^{9}$ Ce faisant,

8 Mohamadou Kane, Roman africain et tradition, Abidjan-Dakar, Nouvelles éditions africaines, 1982.

9 Mohamadou Kane, "Sur les formes traditionnelles du roman africain", Revue de littérature comparée, $\mathrm{n}^{\circ} 48,1974$, p. 536-568 (537). C'est également le point exprimé par Harold Sceub, lors du congrès annuel de l'African Studies Association, en 1984: "There is an unbroken continuity in African verbal art 
86

l'auteur écarte tout rapport de la littérature africaine avec d'autres littératures: "Quel que puisse être, par la suite, son degré d'adaptation à la culture française, il sera toujours impossible d'expliquer ses œuvres en faisant abstraction de son origine. C'est en cela d'ailleurs que réside la différence entre le romancier africain et le romancier étranger qui écrit sur l'Afrique. " 10 Avec Christopher L. Miller, dans Theories of Africans, Francopbone Literature and Antbropology in Africa ${ }^{11}$ la dimension de l'anthropologie différentielle s'accroît au point d'éclipser d'autres méthodes telles la narratologie ou la psychocritique. De ce courant, retenons deux points importants. Privilégiant le contexte historique des cuvres, la critique afrocentriste laisse peu de place à l'analyse des textes et le corpus lui-même est choisi en fonction de la thèse défendue par le critique, alors qu'il existe de nombreuses ouvres qui contredisent les postulats de l'africanité.

Tout en reconnaissant l'existence des motifs du roman européen $^{12}$ dans le roman africain, le deuxième courant de la tendance afrocentriste, en minimise la portée. Son représentant le plus rigoureux sur le plan méthodologique, Amadou Koné, ne précise-t-il pas que "quels que soient les emprunts à la littérature occidentale, ceux-ci ne peuvent pas permettre d'affirmer des liens de filiation entre les textes"13? Si l'on peut dire que le mérite d'Amadou Koné est de s'appuyer sur l'analyse des textes et le processus d'écriture, les résultats auxquels il aboutit témoignent de sa vision idéologique de l'africanité. S'inscrivant dans la perspective de Bakthine, de Goldmann et de Lukacs, Amadou Koné insiste surtout sur "le rôle du contexte historique dans le phénomène de la naissance du genre romanesque " ${ }^{14}$. Certes, une telle méthode permet de montrer dans quelles circonstances histo-

10 Mohamadou Kane, op. cit., p. 549.

11 Christopher L. Miller, Theories of Africans, Francopbone Literature and Antbropology in Africa, Chicago et Londres, The University of Chicago Press, 1990.

12 Par ce terme, emprunté à Milan Kundera, je désigne un type de roman transnational dont l'entreprise historique a commencé à l'aube des Temps Modernes en Europe: "Je ne veux pas dire par là romans créés en Europe par des Européens, rappelle Kundera, mais romans faisant partie de l'histoire de l'Europe [...]”. Cf. Les testaments trabis, Paris, Gallimard, p. 41.

13 Amadou Koné, Des textes oraux au roman moderne. Étude sur les avatars de la tradition orale dans le roman ouest-africain, Franfurt, Verlag fur Interkulturelle Kommunikation, 1993, p. 31.

14 Amadou Koné, op. cit., p. 21. 
riques le roman est né en Afrique, mais comme elle privilégie les seules références à l'africanité alors que celle-ci n'a qu'une valeur idéologique et non une valeur de production des ceuvres, elle laisse le lecteur sur sa faim. En effet, dans la partie réservée à l'analyse détaillée de trois romans - The Healers d'Ayi Kwei Armah ${ }^{15}$, Le devoir de violence de Yambo Ouologuem ${ }^{16}$ et Chaîne de Saidou Bokoum ${ }^{17}$ —, l'auteur ne privilégie que les seules références à la tradition orale, alors que les romans choisis sont structurés plutôt par une intertextualité généralisée incluant autant les références aux récits oraux que les références aux motifs du roman contemporain. Même l'argument de l'unité culturelle de l'Afrique de l'Ouest, que l'auteur donne pour affirmer l'africanité des romans de cette sous-région, mérite d'être nuancé, car, en disant que le lieu et la culture ethnique d'un auteur ne sont pas les seuls éléments déterminants, Amadou Koné n'affirme-t-il pas, par voie de conséquence, qu'un écrivain africain peut utiliser tout aussi bien des motifs et mythes des récits africains ou européens d'autant plus qu'il a été à l'école occidentale ${ }^{18}$. Koné ne reconnaît-il pas que "l'utilisation de la tradition orale dans le roman n'est pas un phénomène nouveau dans l'histoire du genre romanesque ni un procédé spécifique du romancier africain" ${ }^{19}$. Seul le souci idéologique, et par là distinctif, privilégiant les genres "purs " de la littérature orale traditionnelle aux genres "mixtes", "mélangés" voire " hybrides" que révèle le roman africain, explique la double hypothèse de l'auteur:

1. Le roman ouest-africain (probablement, le roman africain en général) est aussi et surtout une composante de la parole artistique traditionnelle dont il s'inspire très souvent et non

15 Ayi Kwei Armah, The healers, Nairobi, East african Publishing House, 1978.

16 Yambo Ouologuem, Le devoir de violence, Paris, Éd. du Seuil, 1968.

17 Saïdou Bokoum, Chaîne, Paris, Denoël, 1974.

18 Amadou Koné cite les exemples d'Amadou Hampaté Bâ qui est peul, mais qui évoque, dans son roman, L'étrange destin de Wangrin ou les roueries d'un interprète africain (Paris, UGE, coll. "10/18", 1973) des divinités et des mythes bambara et de Saidou Bokoum qui est toucouleur, mais exploite les culture malinké et peule. Cf. op. cit., p. 15 et suivantes.

19 Amadou Koné, op. cit., p. 16. À ce propos Jean Peytard a déjà fait une analyse intéressante qui montre que le phénomène de l'oral dans l'écriture romanesque contemporaine est très généralisé, qu'on ne saurait le restreindre à un corpus : "Problèmes de l'écriture du verbal dans le roman contemporain ", La nouvelle critique, numéro spécial : Linguistique et littérature, 1968 , p. 29-34. 
simplement une copie plus ou moins réussie du roman occidental. 2. C'est généralement la littérature traditionnelle africaine qui influence les auteurs africains dans leur tentative d'écrire des romans, car les catégories que l'on dit mal maitrisées par les romanciers africains sont construites selon les modèles de textes traditionnels. ${ }^{20}$

Par ailleurs, en affirmant que le roman africain se trouve au stade où se trouvait le roman européen à la Renaissance, car, dit-il: "personne ne lit Rabelais en pensant à l'esthétique du roman balzacien. Personne ne dit que le roman de Rabelais est mal fait [...] Rabelais écrit le roman français à un stade de son développement " (p. 195), son plaidoyer pour la défense et l'illustration de l'africanité convoque un autre lieu commun discursif: le mythe du "bon sauvage" à civiliser ou à développer. Fort de sa vision téléologique et progressiste, Koné peut annoncer que la maturité du roman africain, à l'instar du roman européen, est liée au progrès économique du continent noir : "Il est probable que progressivement [...] les romanciers de cette partie du monde (l'Afrique) tiendront peut-être plus compte de l'esthétique romanesque occidentale ou plus précisément seront amenés par les mêmes conditions historiques, sociales, culturelles à écrire le roman comme les occidentaux" (p. 196). Ainsi annoncé, le rachat viendra au fur et à mesure que l'écrivain se familiarisera avec les pratiques de l'Occident, mais pour le moment, il doit écrire différemment. De la critique afrocentriste, tirons quatre observations.

1) D'abord, l'expérience de lecture des romans africains permet de dire que de telles études ne sont pas fondées nécessairement sur l'analyse des œuvres, mais sur ce que doit être idéalement un roman africain. Le rêve du roman africain "pur" s'inscrit dans cette logique que permet la définition essentialiste de la littérature africaine. En insistant sur les seules références aux récits de la littérature orale et en ignorant celles du roman européen, Koné, Kane, Jahn, Miller et bien d'autres définissent les règles qui permettent de construire des canons d'un "bon" roman africain. Une telle critique s'inscrit dans, un contexte culturel de l'Afrique, qui commence avec la colonisation, que l'on peut étudier en rapport avec l'histoire des idées.

2) Ces critiques veulent voir tous les romans produits en Afrique - et Dieu sait qu'ils sont très nombreux - dans un seul 
schéma avec ses motifs et ses topoï constants qu'il suffirait de projeter sur chaque ouvre pour vérifier et toujours confirmer son africanité, même si, comme le disait si bien Roland Barthes, à propos des formalistes, "le texte y perd sa différence [...]"21. Ce mythe du "bon sauvage", repris par la critique littéraire, accrédite une vision manichéiste qui voudrait que la décadence - le péché - du roman européen témoigne indéfiniment pour la grâce et la vertu du roman africain ${ }^{22}$. A priori, en effet, pèse sur l'art et le roman européens une présomption de décadence qu'explique un discours critique africain actuel. Sans entrer dans la complexité du discours anthropologique de l'élection du sauvage et ses multiples implications, retenons que l'exaltation et le volte-face des anthropologues réapparaissent presque trait pour trait à la fin des années cinquante comme en témoigne le livre de Janheinz Jahn cité plus haut. En effet, dès le début du $\mathrm{xx}^{\mathrm{e}}$ siècle, le mythe du "bon sauvage", qui s'était transformé en mythe du barbare à civiliser au $\mathrm{xIx}^{\mathrm{e}}$ siècle, est revenu avec les travaux des anthropologues négrophiles comme Frobénius et Delafosse. Alors qu'au XIx ${ }^{e}$ siècle, l'Africain était le sauvage à civiliser, au début du $x^{e}$ siècle, il devient l'homme des origines au contact duquel l'Europe est appelée à se régénérer, du moins en critique littéraire et artistique. Ici et là, on retrouve ce même slogan d'un art à l'état zéro de la pureté originelle. Une nouvelle téléologie travaille le discours critique: le renouveau artistique passe par la rencontre et la reconnaissance de l'originalité africaine. L'africanité, sans que l'on sache exactement ce que cela veut dire, acquiert la fraîcheur d'une chasteté rétinienne. Exalter l'africanité, c'est la poser comme une

21 Roland Barthes, $S / Z$, Paris, Seuil, 1970. L'auteur disait qu'une telle situation conduit le lecteur à un choix incontoumable : "Il faut donc choisir : ou bien placer tous les textes dans un va-et-vient démonstratif, les égaliser [...], les forcer à rejoindre inductivement la Copie dont on les fera ensuite dériver; ou bien remettre chaque texte [...] dans son jeu . avec les autres textes, p. 9.

22 Sur ce malentendu, voir l'analyse de Bernard Mouralis dans L'Europe, l'Afrique et la folie: "Parallèlement, la découverte, au début du $\mathrm{xx}^{e}$ siècle, des arts non européens - notamment arts africains et et océaniens - qui ne relevaient pas jusqu'à lors du champ de l'histoire de l'art allait élargir la réflexion développée à partir de la prise en compte des "primitifs" européens. On appréciait ainsi le vitalisme, voire la barbarie, de ces ouvres exotiques que l'on opposait volontiers à l'asthénie et à l'esprit de décadence qui paraissaient caractériser alors la production occidentale. [...] les cubistes trouvaient d'abord dans l'Art nègre des formes qui les intéressaient parce qu'elles représentaient pour eux des solutions à un grand nombre de problèmes techniques qu'ils se posaient. (p. 70). 
90

contre-valeur exemplaire aux productions culturelles européennes ${ }^{23}$. D'après ces critiques, il existe un fossé qui divise le royaume du salut - l'originalité africaine - du royaume du mal - la dégénérescence de l'art européen - entre lesquels il ne peut y avoir que l'adhésion ou la répudiation. Ces critiques ont non seulement choisi l'Afrique, ses littératures et ses cultures, mais surtout le bon côté de l'histoire de l'écriture et de l'art. C'est ce phénomène que Franz Fanon appelle dans Peau noire, masques blancs (Seuil, 1952) un manichéisme délirant qui consiste à inverser simplement les égalités "Blanc-bon ", "Noir-mauvais" du mythe du "barbare" à civiliser des anthropologues du xIx siècle. La nouveauté africaine est magnifiée selon le schéma chrétien de la chute - l'art européen - et de la Rédemption - l'art africain.

3) En troisième lieu, ce culte farouche de l'originalité africaine développé par la critique va même à l'encontre du projet des écrivains comme en témoigne, entre autres, la réponse de Sony Labou Tansi à la question de savoir si le comportement bizarre de son héros, dans Les yeux du volcan ${ }^{24}$, s'explique par ses origines africaines:

C'est un fou qui n'a pas de couleur. Africain ou Européen, peu importe. Je crois d'ailleurs que nous devons commencer à réfléchir autrement. Jusqu'à présent, nous autres Africains, avons été très archaïques. Les identités ne doivent pas devenir des handicaps. Moi je suis kongo, mais j'ai aussi une capacité d'ouverture sur les autres, la capacité d'accepter les différences. Donc, mon savant n'a pas de couleur. Sa première préoccupation, c'est le travail qu'il fait et non la couleur de sa peau ou la longueur de ses cheveux. ${ }^{25}$

23 Voir à ce propos la critique que Derrida fait au discours anthropologique, surtout à Lévi-Strauss : "La critique de l'ethnocentrisme, thème si cher à l'auteur des Tristes Tropiques, n'a le plus souvent pour fonction que de constituer l'autre en modèle de bonté originelle et naturelle [...]". Cf. De la grammatologie, Paris, Minuit, 1967, p. 167-168. À propos du discours positif tenu à l'égard de l'Afrique et de ses cultures, Wole Soyinka nuance les propos de Derrida: "Tout cela a mené à maints endroits à une quasi-déification de la personne de l'Africain - à cette situation où il fallait que tout Africain fût un prince; cependant, ici encore, cette déification se doublait d'une crainte et d'une répulsion primitives". Cf. Wole Soyinka, Que ce passé parle à son présent, discours de Stockolm lors de la réception de son prix Nobel de littérature, Paris, Belfond, 1986, p. $43-44$.

24 Sony Labou Tansi, Les yeux du zolcan, Paris, Éd. du Seuil, 1988.

25 Sennen Andriamirado, "Sony Labou Tansi, Qu'écrivez-vous?", Jeune Afrique, $n^{\text {os }} 1564-1565$, p. 125 . La critique se chamaille sur l'originalité et la trahison, 
4) Comme l'art d'écrire découle de l'art de lire, l'œuvre de l'écrivain africain résulte pour une bonne part d'un mégatexte transgressant les frontières des genres et des œuvres littéraires ou autres que l'auteur connaît, car les romanciers africains ne lisent pas que les contes de Mamadou et Bineta 26

\section{Critique « européocentriste ", entre l'essai agonique et l'essai cognitif.}

Autant la critique afrocentriste privilégiait les motifs des récits oraux de la tradition orale africaine, autant la critique européocentriste insiste sur la comparaison du roman africain avec les œuvres européennes. Comme pour la première tendance critique, la critique européocentriste connaît deux courants: l'un agonique, l'autre cognitif.

Essentiellement représenté par les travaux de Makhily Gassama $^{27}$, de Keith Waterhouse ${ }^{28}$ et de Jean-Pierre Ndiaye ${ }^{29}$, le premier courant se base sur l'idée selon laquelle il existerait des règles immuables propres au genre romanesque que tout le monde doit respecter (y compris l'écrivain africain) s'il veut écrire un "bon" roman ${ }^{30}$. Ce courant agonique s'oppose aux thèses de l'africanité. Ainsi, analysant le roman de Chinua Achebe, No Longer at Ease, Keith Waterhouse base son jugement à partir de l'esthétique du roman réaliste: "I suppose the heart of the matter is that simplicity is all we ask in the novel [...]. Later when the chronicles of change are more or less complete, some very fortunate writers will be able to fill the framework in, wallowing in the new luxuries of characterisation, motivation, depth, psychology

et se permet même de faire la leçon aux écrivains qui auraient trahi leur propre pratique d'écriture. On ne saurait être plus modeste!

26 Mamadou et Bineta est le titre générique de manuels scolaires de français à l'école primaire coloniale en Afrique française: Afrique occidentale française (AOF) et Afrique équatoriale française (AEF).

27 Makhily Gassama, Kuma, interrogation sur la littérature nègre de langue francaise (roman et poésie), Abidjan-Dakar, Les Nouvelles éditions africaines, 1987.

28 Keith Waterhouse, The New Statesman, LX, septembre 1960, p. 398.

29 Jean-Pierre Ndiaye, "Des tripes et de la créativité ", Jeune Afrique, $\mathrm{n}^{\circ} 718$, 1974 , p. 63.

30 Amadou Koné ( $o p$. cit.) se livre à une décapante et salutaire démystification d'une telle vision européocentriste, démystification qui serait la bienvenue si elle n'était gâtée par un dogmatisme de l'africanité, comme s'il fallait dénoncer un abus pour retomber dans un autre. 
92

and all the rest of it " 31 Cette critique, qui se base sur la "faiblesse" de l'intrigue et la profondeur psychologique, s'adresserait également à beaucoup de romans européens contemporains. Derrière ce discours généralisant se cache un présupposé sournois: le roman africain, à cause de son immaturité, reste encore un genre dont les techniques restent encore non maîtrisées. C'est ce que Jean-Pierre Ndiaye reproche à Saidou Bokoum: "L'élaboration de tout roman suppose que l'on possède ou maîtrise de la technique de l'écriture de la langue dans laquelle on s'exprime [...]. Le roman est toujours une construction, une architecture. Dans Chaîne, le matériau va dans tous les sens" (p. 63). Plus loin le critique reproche au romancier de s'être inspiré de la pire paralittérature occidentale tandis que d'autres voient dans Chaine plutôt l'influence du nouveau roman et du surréalisme ${ }^{32}$.

Comme on le voit, ce courant poursuit a contrario la thèse de l'africanité littéraire des ouvres élaborée par Janheinz Jahn. Imperceptiblement, et sous l'emprise croissante des ouvres, la critique européocentriste glisse d'une valorisation méliorative à la Janheiz Jan sur la nature des ouvres africaines à un jugement excessivement contraire: le roman africain copie les règles du roman européen. Ceux qui, hier, étaient considérés comme les révolutionnaires en art romanesque et manifestaient ainsi une disposition naturelle à l'originalité (Jahn) se sont métamorphosés en imitateurs corrompus par la "mauvaise paralittérature occidentale" (Ndiaye). Le romancier africain est coupable, par avance, pour avoir été déclaré parfait et original par rapport à l'art du roman européen. C'est ici que l'on peut voir que même la critique afrocentriste se réfère au modèle européen du roman. Car le roman africain n'est "original" et "différent" que par rapport au roman européen. S'agissant de la critique européocentriste, le critique convoque l'Âge d'or de l'art pur et naturel perdu par l'Occident et attendu en Afrique pour le révoquer aussitôt. C'est que l'infamie suit la puretê, elle est lui est consubstantielle. Le discours critique débouche alors dans la malédiction et l'injure du plagiat comme

31 Chinua Achebe, No Longer at Ease, London, Heinemann Educational Book, 1963, p. 398. Traduction: we suppose que tout ce que nous demandons au roman, c'est la simplicité [...] Plus tard, quand les changements seront plus ou moins complets, quelques écrivains très chanceux pourront remplir le cadre en plongeant dans les nouveaux délices de la caractérisation, de la motivation, de la profondeur, de la psychologie et tutti quanti.*

Pour plus de détails, voir Amadou Koné, op. cit., p. 176-177. 
chez Yambo Ouloguem, devenu le symbole de la bataille idéologique rangée en deux camps que se livrent les deux tendances de la critique littéraire africaine ${ }^{33}$. Ce discours fonctionne selon un schéma passionnel où le critique projette sur les ouvres africaines un mécanisme amoureux. La première étape de la compréhension était proche du ravissement. Car on explorait avec ivresse l'adéquation inespérée d'un objet à leur désir de révolution artistique (Jahn). La deuxième a été celle du dépit (Ndiaye, Waterhouse). La promotion de la littérature africaine ne saurait dissimuler le fait que la critique européocentriste est, avant tout, amoureuse de sa propre littérature; elle est une projection de l'Occident sur luimême. Car, considérer la littérature africaine comme une compensation à ce qui manque à la littérature européenne, si l'on peut dire ainsi, c'est dégrader la découverte de cette littérature en simple retour à la pureté perdue du "primitivisme" d'avant l'ère moderne, avant le figement des genres littéraires en Europe.

Étudiant les ouvres africaines en rapport avec l'esthétique du roman européen, le deuxième courant s'inscrit dans la tradition critique européenne de la recherche des influences entres les différentes littératures. Ce deuxième courant de la critique européocentriste est typique de l'essai cognitif où les valeurs idéologiques sont posées mais non débattues et où la démonstration n'a qu'une fonction didactique: elle aide à faire comprendre, à dissiper le doute sur la littérarité des ouvres africaines, mais n'est le lieu ni d'une résistance ni d'une attaque de la thèse afrocentriste. Le ton est celui de la démonstration et de l'explication et le critique cherche jusqu'à un certain point à se placer dans une perspective de la littérature universelle. Lilyan Kesteloot a été la première à avoir étudié cet aspect de l'influence du roman européen sur les romanciers africains et antillais dans son célèbre ouvrage, Les écrivains noirs de langue française: naissance d'une littérature ${ }^{34}$. Bernard

33 Quand Le devoir de violence est paru, son auteur reçut le prix Renaudot avant d'être accusé de plagiat. Le procès n'aura pas lieu, mais sa carrière d'écrivain est brisée. Texte complexe, maniant les techniques narratives entrelacées allant de l'intertextualité à la parodie généralisée, ce roman est cité par plusieurs critiques tantôt pour montrer que le roman africain est une continuation de la parole traditionnelle (Koné, Kane et Miller) tantôt pour souligner ses relations avec le roman européen (Mouralis, Kesteloot et julien). Voir aussi l'article de Seth Wolitz, "L'art du plagiat ou une brève défense de Yambo Ouologuem", Research in African Literature, vol. IV, $\mathrm{n}^{\circ} 1,1973$, pp. 130-134.

34 Lilyan Kesteloot, Les écrivains noirs de langue française: naissance dune littérature, Bruxelles, Institut de sociologie de l'Université Libre de Bruxelles, 1963. 
94

Mouralis s'est intéressé aussi à cette question du roman africain dans ses rapports avec les modèles européens ${ }^{35}$. À partir de plusieurs romanciers, allant de Béti à Mudimbe en passant par Yambo Ouologuem et Cheikh Hamidou Kane, les travaux de Mouralis montrent que beaucoup de romans africains adoptent des motifs du roman européen. En montrant, par exemple, comment la folie est un motif dominant dans nombre de fictions africaines, Mouralis renverse le mythe du "bon sauvage", faisant de l'Africain un homme insouciant que la folie ne saurait atteindre. De cette manière, il affirme l'universalité des pratiques culturelles et littéraires de l'Afrique: "Car, vouloir penser l'Autre et se penser à travers la catégorie de la folie et de la maladie, loin d'être l'affirmation d'une singularité, voire d'une stigmatisation, apparaît en définitive comme une inlassable revendication d'appartenance à l'humanité, puisque rien n'est plus humain que la folie et la maladie. " ${ }^{36}$ À l'instar de Paul Van Tieghem, Mouralis inscrit sa démarche critique dans une perspective de compréhension internationale entre les peuples par le biais de la littérature ${ }^{37}$. Dans son ouvrage African Novels and the Question of Orality, Eileen Julien ${ }^{38}$ a fait une analyse globale de la situation en s'attaquant, avec raison, à l'essentialisme de l'africanité et elle a posé un jugement définitif en soulignant, dans la perspective de Todorov ${ }^{39}$, que le roman africain n'est pas une continuation de la parole artistique traditionnelle, car il use, comme d'autres romans, du phénomène

35 Bernard Mouralis, "Le roman africain et les modèles occidentaux", Annales de l'Université d'Abidjan, Série D (Lettres), tome 3, 1970, p. 8-93; "Pour qui écrivent les romanciers africains? Essai de titrologie romanesque ", Présence africaine, vol. 114, 1981, p. 51-78; Les contre-littératures, Paris, Presses universitaires de France, 1975; Mudimbe ou le Discours, l'Écart et l'Écriture, Paris, Présence Africaine, 1988 et L'Europe, l'Afrique et la folie, Paris, Présence africaine, 1993.

36 Bernard Mouralis, L'Europe, l'Afrique et la folie, la quatrième de couverture.

37 Paul Van Tieghem disait, dans sa communication au quatrième congrès international d'histoire littéraire moderne: "La littérature comparée impose [...] à ceux qui la pratiquent, une attitude de sympathie et de compréhension à l'égard de nos frères humains, un libéralisme intellectuel, sans lesquels aucune ouvre commune entre les peuples ne peut être tentée", cité par Pierre Brunel et al., Qu'est-ce que la littérature comparée?, Paris, Armand Colin, 1983, p. 67.

38 Eileen Julien, African Novels and the Question of Orality, Bloomington et Indianapolis, Indiana University Press, 1992.

39 Oswald Ducrot et Tzvetan Todorov, Dictionnaire encyclopédique des sciences du langage, Paris, 1972, p. 446. 
général de l'intertextualité, faisant dialoguer tous les textes entre eux: "We refer to the continuous dialogue of works of literature among themselves as intertextuality: texts comment and expand upon their predecessors or break with them entirely, as their situation is specific social and historical contexts shapes them " ${ }^{40}$. Si cette piste de l'intertextualité est très intéressante et coupe court à des positions idéologiques de l'africanité, Julien tombe dans un autre excès: vouloir étudier les romans africains modernes, qui sont justement caractérisés par une hybridité des formes littéraires et des modalités narratives diverses en termes génériques classiques tels l'épopée, la fable, etc.

Bien que ce courant s'inscrive dans la vision européocentriste de la littérature, la différence avec le premier courant est grande. Mouralis, Kesteloot, Julien et bien d'autres remarquent que le roman africain convoque nombre de motifs littéraires du roman européen, et que, toutes proportions gardées, il s'inscrit dans le champ culturel du roman européen, sans poser un jugement de valeur sur le phénomène lui-même. L’idée générale développée est que les écrivains africains qui connaissent le modèle du roman européen, par la scolarisation et par les moyens de diffusion culturelle internationaux, l'adaptent plus ou moins avec ingéniosité, car, comme le rappelle Milan Kundera, dont je partage le point de vue, "aucun individu ne peut ne pas imiter ce qui a déjà eu lieu " 41 .

\section{Pour une poétique du roman africain}

Après avoir analysé la rhétorique du discours critique littéraire africain appliqué au roman, il s'agit maintenant d'aborder la deuxième partie de ma perspective méthodologique: comment peut-on lire le roman africain eu égard aux différentes positions méthodologiques en cours? Ma démarche s'explique par trois faits majeurs. 1) Dans la mesure où la critique afrocentriste et la critique européocentriste tendent à réduire la liberté créatrice d'un auteur, négliger son originalité pour l'enfermer dans une africanité ou une européanité dont les formes sont répertoriées et

40 P. 26. Traduction: "Nous renvoyons le dialogue continuel d'œuvres littéraires au concept d'intertextualité : les textes commentent, poursuivent le travail de leurs prédécesseurs ou rompent carrément avec eux, selon leur situation sociale spécifique et le contexte historique qui les forme."

Milan Kundera, L'art du roman, p. 22. 
96

fixées à l'avance, de telles positions semblent être une contrainte, non seulement pour les romanciers, mais aussi pour toute critique soucieuse de situer les cuvres africaines dans le contexte culturel du $\mathrm{xx}^{\mathrm{e}}$ siècle, caractérisé par la multiplicité des traits génériques et transculturels. 2) Malgré les apparences, les deux tendances fonctionnent de la même façon. Elles ont, en effet, la même prétention des anthropologues du XIX siècle: le roman européen reste le modèle du roman africain. Elles disent aujourd'hui toujours la même chose, car si la critique afrocentriste se contente de renverser les termes de la vision européocentriste, en affirmant l'africanité des textes, la critique européocentriste poursuit allègrement la ligne tracée depuis le $\mathrm{xtx}^{\mathrm{e}}$ siècle. En répondant au présupposé de la critique européocentriste, la critique afrocentriste affirme, par exemple, que le roman africain est original parce qu'il n'emprunte pas ses motifs au roman européen, mais à la parole artistique de la tradition orale. Même si tel était le cas, en quoi le fait de se référer à la parole artistique de la tradition orale constitue-t-il un effet valorisant de l'écriture romanesque sinon le fait que le discours critique prend sa position à partir de l'essentialisme de la critique européocentriste pour en renverser les termes? 3) Dès lors que ces critiques se font des critères africains ou européens du roman, aussi parfaits par leur originalité que par leur beauté tels qu'on ne les retrouve pas dans les ceuvres, ils dénient aux écrivains le droit d'écrire librement. Ce faisant, ils refusent l'héritage culturel complexe du romancier moderne alors que le thème de la différence, ici l'africanité et l'européanité, est par excellence, un thème girouette qui n'a aucune vérité en lui-même en littérature puisqu'il tourne au gré des idéologies et témoigne de l'ambivalence de certaines valeurs susceptibles d'être invoquées à propos d'attitudes très opposées.

De ces considérations se dégagent quelques éléments notables pour la lecture du roman africain. 1) Le roman africain est, comme le roman contemporain, caractérisé par un mélange de genres. Il n'est ni une copie du roman "Occidental" ni une continuation de la parole artistique traditionnelle. 2) Si les traits génériques des récits oraux de la littérature orale africaine sont une dominante dans certains textes, c'est loin d'être le cas dans d'autres romans, comme en témoignent les grands classiques africains: Mongo Beti, Ousmane Sembène, Cheik Hamidou Kane, Valentin-Yves Mudimbe, Sony Labou Tansi, Henri Lopès et bien d'autres. Au lieu de chercher uniquement les références à la tradition orale ou les influences du 
roman européen, il vaut mieux mettre les textes en relation avec le genre romanesque afin de voir comment celui-ci transforme, réutilise d'autres genres littéraires et les intègre à ses propres règles. 3) Réfléchir sur le fait que le roman africain participe de l'esthétique du roman contemporain a l'avantage de replacer les romans africains dans l'histoire du roman, celle de la rencontre des genres des littératures de l'Afrique et celles de l'Europe. Car, les formes du roman africain, pour ainsi dire, convoquent celles du roman et celles d'autres genres littéraires même si les contenus peuvent diffêrer suivant les thèmes développés répondant davantage aux préoccupations idéologiques qu'aux dimensions esthétiques. Sinon comment peut-on expliquer autrement la sève de type rabelaisien qui nourrit tant de romans africains? 4) Une telle perspective est d'autant plus importante que continuer à chercher les spécificités du roman africain débouche sur une aporie parce que la vie culturelle du $\mathrm{xx}^{\mathrm{e}}$ siècle, caractérisée par plusieurs modes et moyens de communication mettant en contact diverses formes d'expression culturelle et la capacité du genre romanesque à transformer et à phagocyter toutes les formes artistiques contemporaines, permet difficilement que l'on puisse imaginer l'existence des formes spécifiques au roman national: africain, français, latino-américain ou québécois ${ }^{42}$. 5) L'inscription des références aux éléments esthétiques de l'oralité et leur mise en narration romanesque ainsi que l'adaptation des démarches esthétiques contemporaines constituent des éléments constitutifs de l'émergence de la modernité romanesque africaine. Sous cet angle, la littérarité des textes africains n'est plus à chercher dans la spécificité "africaine" ou dans l'influence "européenne" mais dans tous les effets de sens à reconstruire en relation avec la notion de genre littéraire et la façon dont celle-ci se présente comme consubstantielle à l'écriture littéraire elle-même. Découvrir cette modernité, dont la caractéristique majeure est l'hybridité synthétique des formes esthétiques de la tradition africaine, du roman européen et d'autres genres artistiques comme le cinéma et le théâtre, tel est l'enjeu majeur qui devrait être à la base de la critique littéraire africaine.

42 On peut, en effet, considérer le champ culturel mondial, avec ses moyens de communication et de transport qui sont les plus perfectionnés depuis que l'homme est sur la terre, comme un ensemble macrosémiotique expliquant selon quels mécanismes et selon quelles lois les productions culturelles élaborées dans un lieu donné du globe collaborent à sa propre structuration et à son propre développement et à la structuration et au développement de l'ensemble. 